\title{
Volcanogenic Dark Matter and Mass Extinctions
}

\author{
Samar Abbas *\# and Afsar Abbas \# \\ \# Institute of Physics, Bhubaneswar-751005, India \\ * Physics Dept., Utkal University, Bhubaneswar-751004, India \\ (e-mail : abbas@iopb.ernet.in, afsar@iopb.ernet.in)
}

\begin{abstract}
The passage of the Earth through dense clumps of dark matter, the presence of which are predicted by certain cosmologies, would produce large quantities of heat in the interior of this planet through the capture and subsequent annihilation of dark matter particles. This heat can cause large-scale volcanism which could in turn have caused the extinction of the dinosaurs and other mass extinctions. The periodicity of such volcanic outbursts agrees with the frequency of palaeontological mass extinctions as well as the observed periodicity in the occurrence of the largest flood basalt provinces on the globe.
\end{abstract}


While investigating the possibility that a certain weakly interacting massive particle (WIMP), the cosmion, could explain both the dark matter (DM) problem and the solar neutrino problem, Press and Spergel [ 1 ] estimated the rate at which the Sun or a planet will capture WIMPs. For the Earth the capture rate is, as given by Krauss et al [2 ]:

$$
\dot{N}_{E}=\left(4.7 \times 10^{17} \sec ^{-1}\right)\{3 a b\}\left[\frac{\rho_{0.3} \sigma_{N, 32}}{\bar{v}_{300}^{3}}\right]\left(\frac{1}{1+m_{X}^{2} / m_{N}^{2}}\right)
$$

where $m_{X}$ is the mass of the DM particle, $m_{N}$ is the mass of a typical nucleus off which the the particle elastically scatters with cross-section $\sigma_{N}$, $\rho_{X}$ is the mean mass density of DM particles in the Solar System, $\bar{v}$ is the r.m.s. velocity of dark matter in the Solar System, $\rho_{0.3}=\rho_{X} / 0.3 \mathrm{GeV} \mathrm{cm}^{-3}$, $\sigma_{N, 32}=\sigma_{N} / 10^{-32} \mathrm{~cm}^{2}, \bar{v}_{300}=\bar{v} / 300 \mathrm{kms}^{-1}$, and $a$ and $b$ are numerical factors of order unity which depend on the density profile of the Sun or planet.

The Earth will continue to accrete more and more particles until their number density inside the planet becomes so high that they start to annihilate. This could lead to a flux of neutrinos [2-4].

Depending on the nature of the dark matter ( neutralino, photino, gravitino, sneutrino, Majorana neutrino, etc. ), different annihilation channels are possible [3-5 ]. Generally the most significant channels are $\chi \bar{\chi} \rightarrow q \bar{q}($ quark-antiquark ), $\chi \bar{\chi} \rightarrow \gamma \gamma$ ( photons ) and $\chi \bar{\chi} \rightarrow l \bar{l}$ ( lepton-antilepton ). In the quark channel hadronization will take place through jets and subsequent radiative decay will lead to mesons which in turn will decay through their available channels. Hence [5]:

$$
\chi \bar{\chi} \rightarrow q \bar{q} \rightarrow\left(\pi^{0}, \eta, \ldots\right) \rightarrow \gamma+Y
$$

All annihilation processes which directly or indirectly create photons and where energy is delivered to the core through inelastic collisions would lead to the generation of heat in the core. We wish to study this heat generation in the Earth's core through annihilation. This heat is :

$$
Q_{E}=e \dot{N}_{E} m_{X}
$$

where e is the fraction of annihilations which lead to the generation of heat in the core of the Earth. Note that e may be as large as unity for the ideal case where the WIMPs annihilate predominantly through photons only. We shall however take it to be $\sim 0.5[3-5]$ for an order of magnitude estimate. 
Taking $a b \sim 0.34[2], \rho_{0.3}=1, \bar{v}_{300}=1, m_{X}=55 G e V$ and the crosssection on iron to be $\sigma_{N}=10^{-33} \mathrm{~cm}^{2}$, we find that $\sim 10^{8} \mathrm{~W}$ of heat is generated.

This estimate for the heat generation in the core of the earth is for an isotropic DM distribution. However, clumpiness may be a generic characteristic of CDM ( cold dark matter ) cosmologies. Recently it has been argued that in the cosmic string, texture and inflationary models CDM clump cores are formed with core densities given by $\rho_{c} \sim 4.5 \times 10^{8} \rho_{X} \Omega^{2} \times h^{4}[6,7]$. These cores occur over a a smooth background halo density $\rho_{X}$. Although the core densities can exceed the local halo density by a factor of $10^{10}[7]$, one may take the conservative value of $\rho_{c} \sim 10^{8} \rho_{X}$. These clumps are expected to cross earth with a periodicity of 30-100 Myrs [ 8 ]. Thus during the passage of the Earth through such clumps at regular intervals, the flux of the incident DM particles will increase by roughly a factor of $\sim 10^{8}$. Consequently the value of $\dot{Q}_{E}$ during the passage of a clump will be $\sim 10^{16} \mathrm{~W}$.

Improving upon the previous work, Gould [ 9 ] obtained greatly enhanced capture rates for the Earth ( 10-300 times that previously believed ) when the WIMP mass roughly equals the nuclear mass of an element present in the Earth in large quantities, thereby constituting a resonant enhancement. Gould's formula gives the capture rate for each element in the Earth as [9] :

$$
\dot{N}_{E}=\left(4.0 \times 10^{16} \sec ^{-1}\right) \bar{\rho}_{0.4} \frac{\mu}{\mu_{+}^{2}} Q^{2} f\left\langle\hat{\phi}\left(1-\frac{1-e^{-A^{2}}}{A^{2}}\right) \xi_{1}(A)\right\rangle
$$

where $\bar{\rho}_{0.4}$ is the halo WIMP density normalized to $0.4 G e V \mathrm{~cm}^{-3}, \mathrm{Q}=\mathrm{N}$ - $\left(1-4 \sin ^{2} \theta_{W}\right) \mathrm{Z} \sim \mathrm{N}-0.124 \mathrm{Z}, \mathrm{f}$ is the fraction of the Earth's mass due to this element, $A^{2}=\left(3 v^{2} \mu\right) /\left(2 \hat{v}^{2} \bar{\mu}_{-}\right), \mu=m_{X} / m_{N}, \mu_{+}=(\mu+1) / 2$, $\mu_{-}=(\mu-1) / 2, \xi_{1}(A)$ is a correction factor, $v=$ escape velocity at the shell of Earth material , $\hat{v}=3 k T_{w} / m_{X}=300 \mathrm{kms}^{-1}$ is the velocity dispersion, and $\hat{\phi}=v^{2} / v_{\text {esc }}^{2}$ is the dimensionless gravitational potential.

In the WIMP mass range $15 \mathrm{GeV}-100 \mathrm{GeV}$ this yields total capture rates of the order of $10^{17} \mathrm{sec}^{-1}$ to $10^{18} \mathrm{sec}^{-1}$. According to the equation above, this yields $Q_{E} \sim 10^{8} \mathrm{~W}-10^{10} \mathrm{~W}$ for a uniform density distribution.

In the case of clumped DM with core densities $10^{8}$ times the galactic halo density, global power production due to the passage of the Earth through a DM clump is $\sim 10^{16} W-10^{18} W$. It is to be noted that this heat generated 
in the core of the Earth is huge and arises due to the highly clumped CDM.

Within the geothermodynamic theory it is believed that continuous heat absorption by the D" layer ( the lowermost layer of the mantle ) would result from a temporary increase in heat transfer from the core [ 10 ]. This process is thought to continue until, due to its decreasing density, this layer becomes unstable, eventually breaking up into rising plumes ( plume production being an efficient way of heat transfer ). This viewpoint of a lower mantle origin for plumes is strengthened by several recent observations. Firstly, high levels of He-3 reported for Siberian flood basalts [ 11 ] support this view, primordial He-3 having been retained within the lower mantle. Secondly, high levels of Osmium-187 ( a decay product of the element Rhenium-187 which is thought to exist in high concentrations in the Earth's core ) observed in Siberian flood basalts [ 12 ] suggest that some of these rocks may even come from the outer core.

A typical plume created in this manner would, due to its lower density, well upwards. In this process, decompression of the plume on account of its ascent in a pressure gradient will lead to partial melting of the plume head, thereby producing copious amounts of basaltic magma [ 13 ]. Mantle velocities being $\sim 1 \mathrm{~m}$ /year, such a plume would take $\sim 5$ million years to reach the crust. It would then melt its way through the continental crust, thereby producing viscous acidic ( silicic) magma [ 13 ].

The ultimate arrival of such a plume head at the surface could be cataclysmic. Initial explosive silicic volcanism would be followed by periods of large-scale basalt volcanism that ultimately lead to the formation of massive flood basalt provinces such as the Siberian Traps, the Deccan Traps in India and the Brazilian Paraná basalts. Extensive atmospheric pollution would follow; the Deccan Trap flood basalt volcanic episode ( $\sim 65$ million years ago ) ejected an estimated total of $11 \times 10^{6} \mathrm{~km}^{3}$ of basalt, $17 \times 10^{12}$ tonnes of $\mathrm{H}_{2} \mathrm{SO}_{4}, 27 \times 10^{10}$ tonnes of $\mathrm{HCl}$, and $19 \times 10^{3} \mathrm{~km}^{3}$ of fine dust [ 14 ]. Climatic models predict that this is capable of triggering a chain of events ultimately leading to the depletion of the ozone layer, global temperature changes, acid rain and a decrease in surface ocean alkalinity.

Thus, Deccan volcanism has been proposed as a possible cause for the $\mathrm{K} / \mathrm{T}$ ( Cretaceous/Tertiary ) mass extinction that extinguished the dinosaurs $[13,15]$, while the Siberian basalts have been put forth as a possible culprit for the $\mathrm{P} / \mathrm{T}$ ( Permian/Triassic ) mass extinction [ 12 ]. In fact, there exists a striking concordance between the ages of several major flood basalt provinces 
and the dates of the major palaeontological mass extinctions [ 10 ].

It was shown above that the passage of the Earth through a DM clump core could produce $\sim 10^{16}-10^{18} \mathrm{~W}$ of heat. The annual heat flux of the Earth amounting to $4.2 \times 10^{13} \mathrm{~W}[16$ ], this would have significant geothermodynamical effects which future work shall shed more light on. Here, however, we suggest that this large excess of heat produced in the interior of the Earth could lead to violent volcanism and mass extinctions via the process elucidated above.

In a recent paper, Collar set forth the hypothesis [ 8 ], that dark matter induces cancers in organisms, thereby causing mass extinctions. This hypothesis fails to explain several features associated with the mass extinction record. The iridium anomaly, the occurrence of soot, spherules, and other features at the $\mathrm{K} / \mathrm{T}$ boundary generally attributed either to an impact or a massive volcanic outburst [ 17,18] find no explanation within this scenario. Moreover, sea level regression thought to occur at several mass extinctions cannot fit in this model [ 8 ]. The occurence of several large flood basalt provinces with ages concordant with several mass extinctions cannot be visualized therein [8].

The volcanic hypothesis, despite providing a viable explanation for several features reported for mass extinctions, has always lacked a compelling reason for otherwise supposedly haphazard eruptions to occur in a periodic fashion. When one takes into account that the Earth has been cooling ever since its formation ( which implies a consequent decrease in volcanic activity), this objection becomes a serious weakness. It is hoped that a viable reason for large volcanic eruptions to occur in a periodic manner has been presented here with the introduction of the volcanogenic dark matter scenario. This should strengthen the volcanic hypothesis of mass extinctions.

Correspondence and requests for materials to either authors Samar Abbas; e-mail : abbas@iopb.ernet.in

Afsar Abbas; e-mail : afsar@iopb.ernet.in 


\section{References}

1. Press W. H. and Spergel D. N., Ap. J. 296, 679-684 (1985)

2. Krauss L. M., Srednicki M. and Wilczek F., Phys. Rev. D33, 2079$2083(1986)$

3. Gaisser T. K., Steigman G. and Tilav S., Phys. Rev. D34, 2206-2222 (1986)

4. Freese K., Phys. Lett. B167, 295-300 (1986)

5. Bengtsson H-U., Salati P. and Silk J., Nucl. Phys. B346, 129-148 (1990)

6. Silk J. and Stebbins A., Ap. J., 411, 439-449 (1993)

7. Kolb E. W. and Thachev I. I., Phys. Rev.D50, 769-773 (1994)

8. Collar J. I., Phys. Lett. B368, 266-269 (1996)

9. Gould A., Ap. J. 321, 571-585 (1987)

10. Courtillot V. E., Sc. Am., 85-92 (Oct. 1990)

11. Basu A. R., Poreda R. J., Renne P. R., Teichmann F., Vasilev Y. R., Sobolev N. V. and Turrin B. D., Science 269, 822-825 (1995)

12. Walker R. J., Morgan J.W., Horan M.F., Science 269, 819-822 (1995)

13. Campbell I. H., Czamanske G. K., Fedorenko V. A., Hill R. I. and Stepanov V., Science 258, 1760-1763 (1992)

14. Officer C. B.,Hallam A., Drake C. L. and Devine J. D., Nature 326, 143-149 (1987)

15. Officer C. and Page J., " The Great Dinosaur Controversy ", AddisonWesley (1996)

16. Vacquier V., Geophys. J. Int. 106, 199-202 (1991)

17. Bhandari N., Current Sc. (India) 61, 97-104 (1991)

18. Sutherland F. L., Earth-Sc. Rev. 36, 1-26 (1994) 